\title{
A Wireless Sensor for Ingot Mold Temperature Monitoring
}

\author{
S. Mamaschew*, I. Hüllen**, J. Schlüter**, M. Vossiek* \\ ${ }^{*}$ Clausthal University of Technology, Institute of Electrical Information Technology \\ ** SMS Siemag AG, Düsseldorf
}

\begin{abstract}
In order to avoid ruptures of a metal strand in continuous casting machines a matrix of numerous temperature sensors is applied to monitor the temperature profile of the strand. The need for frequent reconstruction and maintenance of the ingot mold leads to efforts with respect to the cabling of the numerous sensors. In this paper a prototype of a completely wireless thermoelectrically powered temperature sensor is introduced. The solution includes the energy-harvesting and sensor electronics and a robust sensor design. The approach of the dimensioning is described and the relevant energy harvesting components are characterized in the trial. Measurements show that the developed thermoelectric powered sensor can transmit every second up to 28 bits of user data in addition to a 32 bit unique sensor ID if a temperature difference of $16{ }^{\circ} \mathrm{C}$ between the sensors hot surface and the ambient air is given.
\end{abstract}

\section{Introduction}

A rupture of a metal strand is one of the most severe disruptions that can happen during steel production. To avoid this accident, it is essential to measure the temperature profile of the metal strand in order to identify the development of cracks [1]. For this task a matrix of up to 120 spatially distributed temperature sensors is used. The setup of a typical continuous casting plant is depicted in Fig. 1. The sensor matrix is located at the inner side of the ingot mold that is oriented towards the molten metal strand. The cabling of the numerous sensors causes notable problems with respect to installation efforts and reliability. In addition, if the ingot mold needs to be re-arranged, the required adjustment of the cabling leads to long and thus costly downtimes.

To overcome this problem, a concept for a wireless sensor system has been developed. It is proposed that a thermoelectric generator (TEG) utilizes the

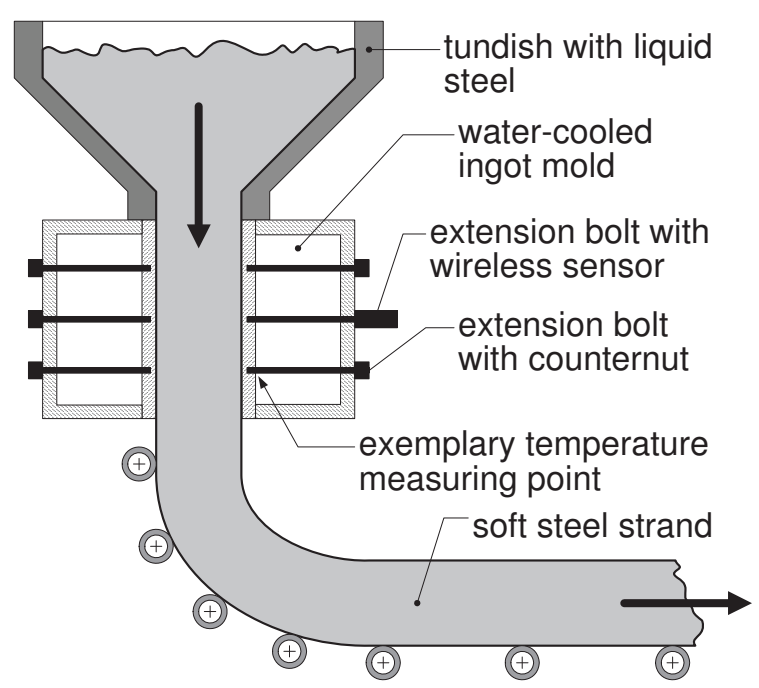

Fig. 1: Soft steel strand process heat of the ingot mold as an energy source. The sensor is screwed into the hot mold wall, so that the absorbed heat can pass through the TEG mounted in the interior of the sensor to the ambient air. The temperature is measured with a sensing element in a probe that is pressed against the inner side of the ingot mold. The extreme high humidity, pollution by settled dust, the potential mechanical forces, and the need for an easy installation and handling were considered in the sensor design. As usually the electronic part of the energy harvesters consists of an energy converter, an energy conditioner, and an energy storage (see Fig. 2). The storage supplies a microcontroller that manages the supply and the data conversion for the transducer and the RF transmitter module. A simple low power transmission protocol with a low probability of collision is used for the radio transmission.

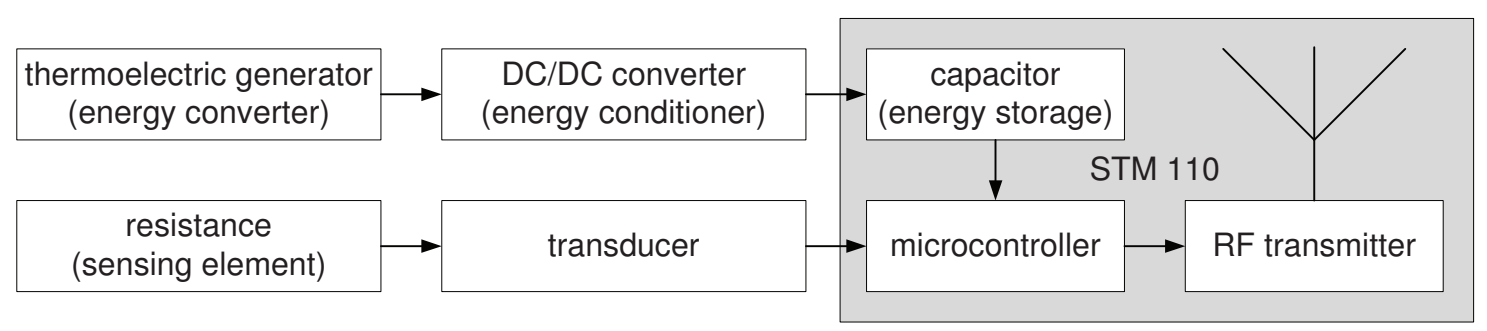

Fig. 2: Schematic of the sensor electronics 


\section{Design of the Thermoelectric Energy Converter}

For a suitable choice of the energy converter several Seebeck elements were investigated. Their parameters were determined experimentally. The results are summarized in Tab. 1. To ensure efficient energy conversion the modules need to have a high Seebeck coefficient, a low internal resistance, and a low thermal conductivity. Since all parameters rise with the number of thermocouples it is possible to increase the thermoelectric efficiency [2] by optimizing the material composition of the used thermocouples. A preferred material composition is characterized by a highest possible figure of merit [2], as in the example of the TEG 1. Other manufacturers rely on temperature resistant and mechanical robust modules such as the TEG 2. However, because of the used polyimide insulation between the thermocouples in order to stabilize the structure, these modules are attended by a loss of the thermoelectric efficiency. Also the TEG 3 does not has a high figure of merit, but considering its small surface an excellent power factor [2]. The TEG 3 is an extremely miniaturized module, based on a novel production technology. Depending on the restrictions of the application (for example weak heat source, high temperature or low space) the type of the TEG should be selected. The bold parameters in the table show the main highlight of each TEG type.

Tab. 1: Measured parameters of tested Seebeck elements

\begin{tabular}{|l|l|l|l|}
\hline description & TEG 1 & TEG 2 & TEG 3 \\
\hline manufacturer & Eureca & HI-Z & Micropelt \\
\hline model & TEG1-30-30-2.1 & $\mathrm{HZ}-2$ & MPG-D751 \\
\hline maximum temperature & $150{ }^{\circ} \mathrm{C}$ & $\mathbf{2 5 0}{ }^{\circ} \mathbf{C}$ & $200{ }^{\circ} \mathrm{C}$ \\
\hline area & $900 \mathrm{~mm}^{2}$ & $841 \mathrm{~mm}{ }^{2}$ & $13.9 \mathrm{~mm}$ \\
\hline height & $3.6 \mathrm{~mm}$ & $5.1 \mathrm{~mm}$ & $1.1 \mathrm{~mm}$ \\
\hline number of thermocouples & 128 & 98 & 540 \\
\hline Seebeck coefficient & $34.1 \mathrm{mV} / \mathrm{K}$ & $18.3 \mathrm{mV} / \mathrm{K}$ & $99 \mathrm{mV} / \mathrm{K}$ \\
\hline internal resistance & $6.5 \mathrm{Ohm}$ & $3.4 \mathrm{Ohm}$ & $524.6 \mathrm{Ohm}$ \\
\hline thermal conductivity & $0.23 \mathrm{~W} / \mathrm{K}$ & $0.33 \mathrm{~W} / \mathrm{K}$ & $0.15 \mathrm{~W} / \mathrm{K}$ \\
\hline figure of merit & $\mathbf{7 7 9 1 / K}$ & $3021 / \mathrm{K}$ & $1241 / \mathrm{K}$ \\
\hline power factor & $4.97 \mu \mathrm{W} /\left(\mathrm{K}^{2} \cdot \mathrm{cm}^{2}\right)$ & $2.93 \mu \mathrm{W} /\left(\mathrm{K}^{2 \cdot} \mathrm{cm}^{2}\right)$ & $\mathbf{3 2 . 6 8} \boldsymbol{\mu W} /\left(\mathrm{K}^{\mathbf{2}} \cdot \mathrm{cm}^{2}\right)$ \\
\hline
\end{tabular}

When choosing the appropriate energy converter, the thermal sensor assembly and the environmental conditions must be considered as well. This includes the absorption and dissipation of the heat, as well as the arrangement and mounting of the TEG. The hot side has to provide enough heat and the cold side has to release enough heat to produce a sufficiently large and durable temperature difference for the TEG. A simplified arrangement with an infinite heat source and a heat sink with a finite energy transfer are shown in Fig. 3.

With the hot side temperature $T_{0}$ and the ambient temperature $T_{2}$ the temperature difference at the TEG can be calculated
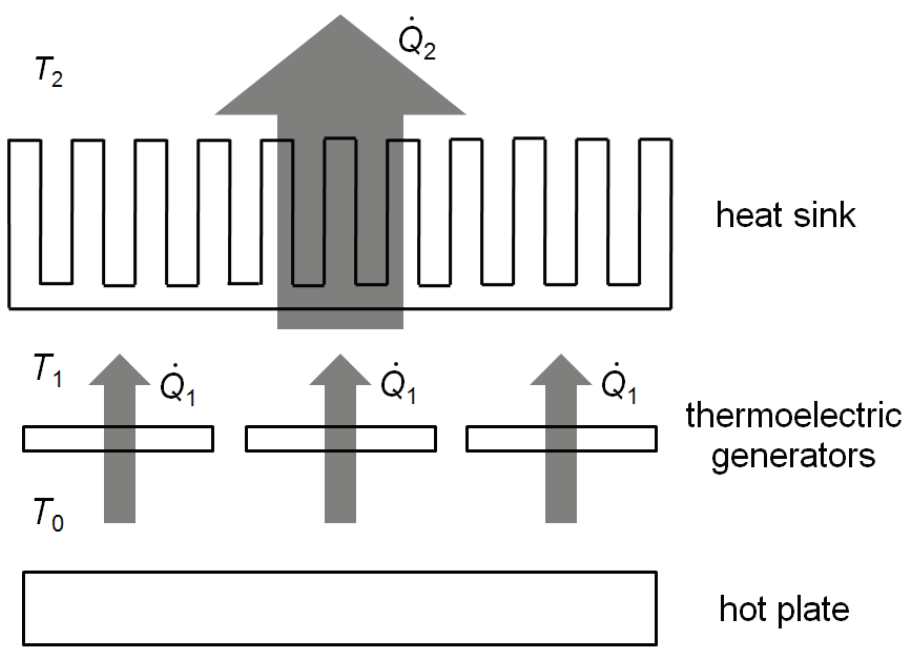

Fig. 3: Parallel array of thermoelectric generators by

$$
\Delta T_{T E G}=T_{1}-T_{0}=\frac{G_{K}\left(T_{0}-T_{2}\right)}{n G_{T}+G_{K}}=\frac{G_{K}\left(T_{H}-T_{C}\right)}{n G_{T}+G_{K}}
$$


where $G_{K}$ represents the thermal conductivity of the heat sink and $G_{T}$ the thermal conductivity of a single TEG [3]. The losses of the thermal coupling and other thermal bridges are neglected in this approach. The number of the TEGs $n$ reduces the effective temperature difference due to the additional heat flows $\dot{Q}_{1}$ with the result that the heat sink heats up to the temperature $T_{1}$. Assuming identical modules with a Seebeck coefficient $\alpha$ the thermoelectric voltage $U_{0}$ at each TEG is given by

$$
U_{0}=\alpha \cdot \Delta T_{T E G} .
$$

Under consideration of the internal resistance $R_{I N}$ the maximum output power is described by

$$
P_{\text {max }}=\frac{1}{4} \frac{U_{0}{ }^{2}}{R_{I N}} \cdot n=\frac{\alpha^{2} \cdot \Delta T_{T E G}{ }^{2}}{4 \cdot R_{I N}} \cdot n=\frac{n \cdot \alpha^{2} \cdot G_{K}{ }^{2} \cdot\left(T_{H}-T_{C}\right)^{2}}{4 \cdot R_{I N} \cdot\left(n \cdot G_{T}+G_{K}\right)^{2}} .
$$

In order to maximize the output power the thermal conductivity of the heat sink has to be adjusted depending on the number of TEGs and its thermal conductivity according to:

$$
G_{K}>\sqrt{n} \cdot G_{T}
$$

The increase of the number of the TEGs must reasonably be accompanied by a better heat dissipation of the heat sink. The recommendation respective the thermoelectric voltage looks different. When the energy converters are connected in series the thermoelectric voltage increases with the number of TEGs always despite the raised heat flux. With the disadvantage of reduced overall performance this option would be possible (for example at very small temperature differences) in order to stay still in the operating range of the voltage converter.

\section{Configuration and Assembling of the Prototype}

A multi-part housing made of brass was designed for the prototype as shown in Fig. 4. The sensor head includes 4 TEGs from Micropelt (type TEG 3 in Tab. 1), which are installed between the cold side and hot side of the sensor. The TEGs are stored in a floating bearing with a well heat-conducting low-melting metal alloy (In-Ga alloy) in a gap between the housing halves. It is used a guide bush (to take up the radial forces) and a spacer (for receiving the axial forces) to protect the relatively small and sensitive TEGs. Both housing halves are clamped by a screw (with a through hole for probe and cable).

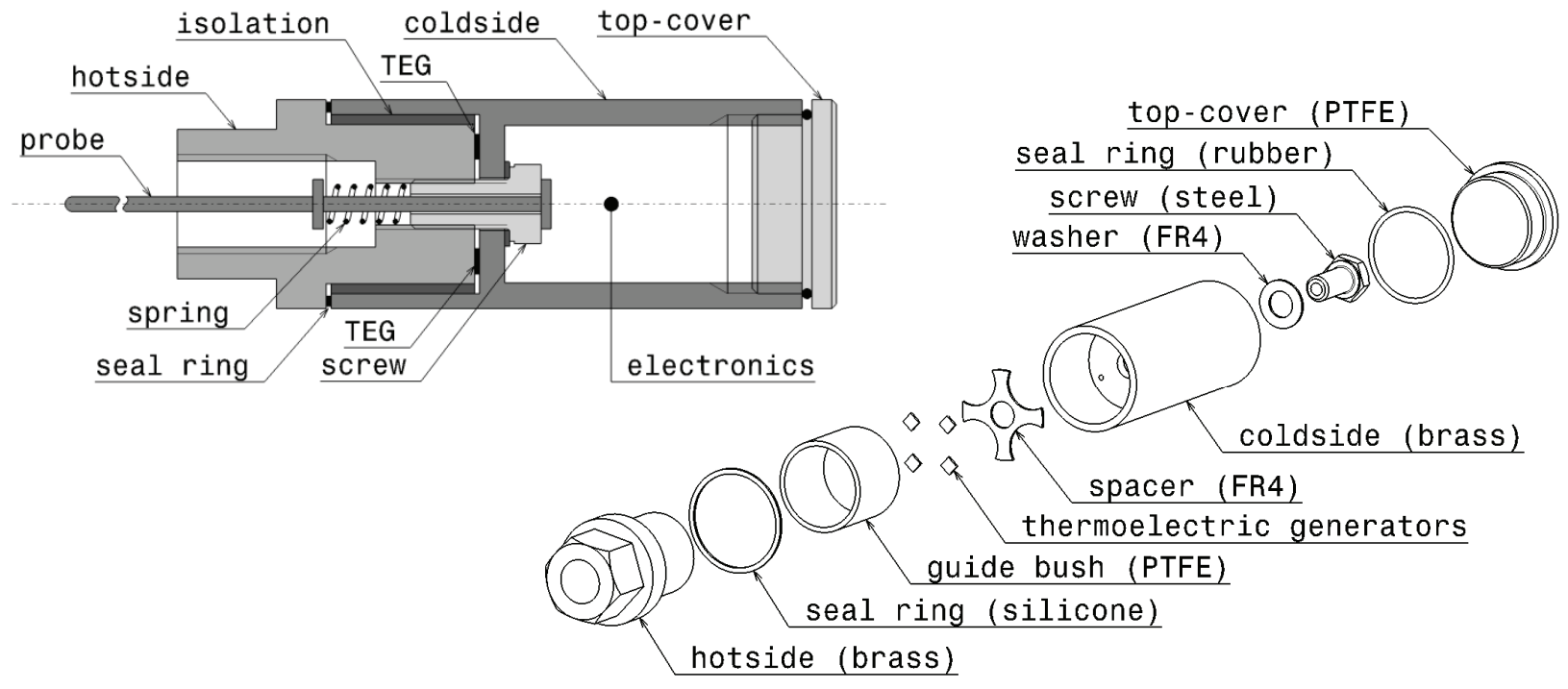

Fig. 4: Assembling of the thermoelectric powered sensor 
To generate power the sensor head is first screwed to a bolt in the mold wall (see Fig. 5). Then the heat flow from the ingot mold is conducted through the TEGs and dissipated by a cylindrical surface with $125 \mathrm{~cm}^{2}$. Due to the given extreme dust deposits, cooling fins would be blocked very fast and thus they cannot be used to increase the heat transfer. To reduce thermal bridges, the mechanical contacts between cold side and hot side (guide bush, spacer, and washer) are made of plastic. As recommended in equation (4), the heat sink was designed with a thermal conductivity of $0.4 \mathrm{~W} / \mathrm{K}$. At a temperature difference of $10^{\circ} \mathrm{C}$, it is expected (according to equations (2) and (3)) to gain a thermoelectric voltage of $400 \mathrm{mV}$ for each TEG and a total power output of $1.2 \mathrm{~mW}$.

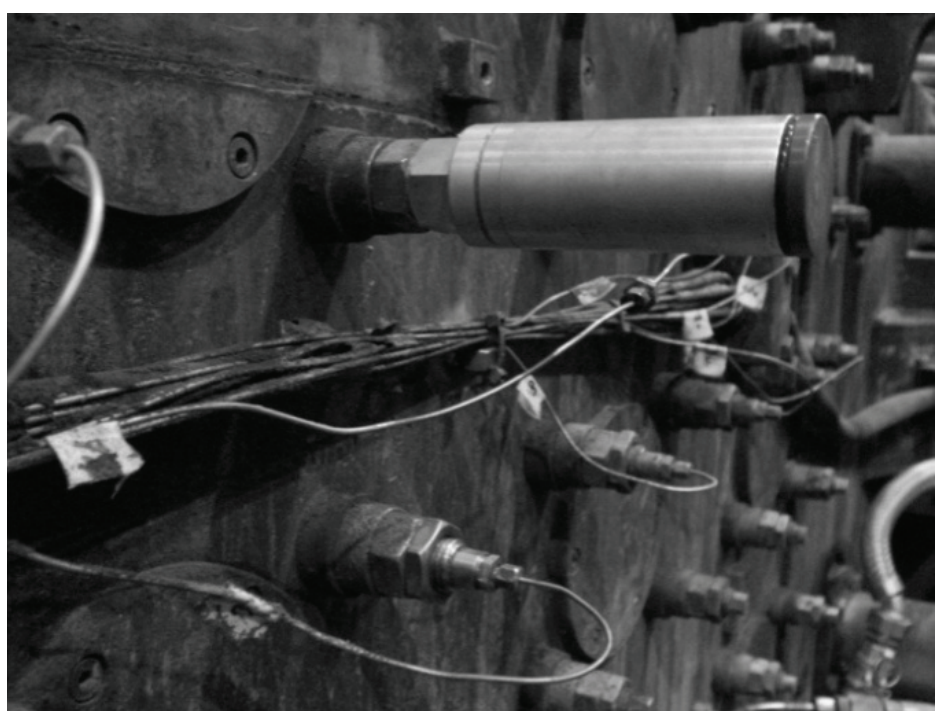

Fig. 5: Installed sensor at the ingot mold

To convert and stabilize the low thermoelectric voltage to a sufficiently high level for the electronics a voltage converter of Micropelt is used. This DC/DC converter was chosen because of the relatively high (for energy harvesting applications) expected input voltage. With increasing the input voltage, in general, the usage of a converter with higher efficiency is possible [4]. The selected boost converter operates on the principle of mutual induction and can achieve an efficiency of up to $73 \%$. It was measured that the boost converter required an input voltage of $300 \mathrm{mV}$ at $26 \mu \mathrm{W}$ power consumption to produce an output voltage of $3.3 \mathrm{~V}$.

The generated energy is stored in a capacitor of $100 \mathrm{mF}$ on the EnOcean STM110. A power management controls the power supply for transducer, microcontroller, and RF transmitter. A temperature dependent resistance in the tip of a spring-mounted probe is used as sensing element. The transducer itself is temperature compensated and uses the 8-bit A/D converter in the microcontroller as an interface. For energy saving purposes, the RF transmitter is turned on only when a significant change of the temperature is measured, and only during data transmission.

The radio module works on the $868.3 \mathrm{MHz}$ band with a maximum output of $10 \mathrm{~mW}$ at a data rate of 125 kbps (amplitude-shift keying). To reduce the probability of collision a proprietary protocol [5] was used with a length of about $1.2 \mathrm{~ms}$ for one radio telegram. In addition to the 28 bit user data and the 32 bit ID the protocol includes 40 bit overhead (for synchronization, header, and checksum). The antenna of the radio module is fixed directly behind the plastic top-cover of the sensor head. An installed receiver in line of sight of the ingot mold passes the received data to a computer for further evaluations.

As part of preliminary tests to verify the suitability of the selected sensor, the RF electronics, and the mechanical design an additional prototype with battery supply has been developed. The prototype has the same electrical and mechanical design (see Fig. 6) as the sensor described before, except for the voltage converter. For the power supply 3 cells per $1.5 \mathrm{~V}$ of the type LR61 (AAAA) were selected.

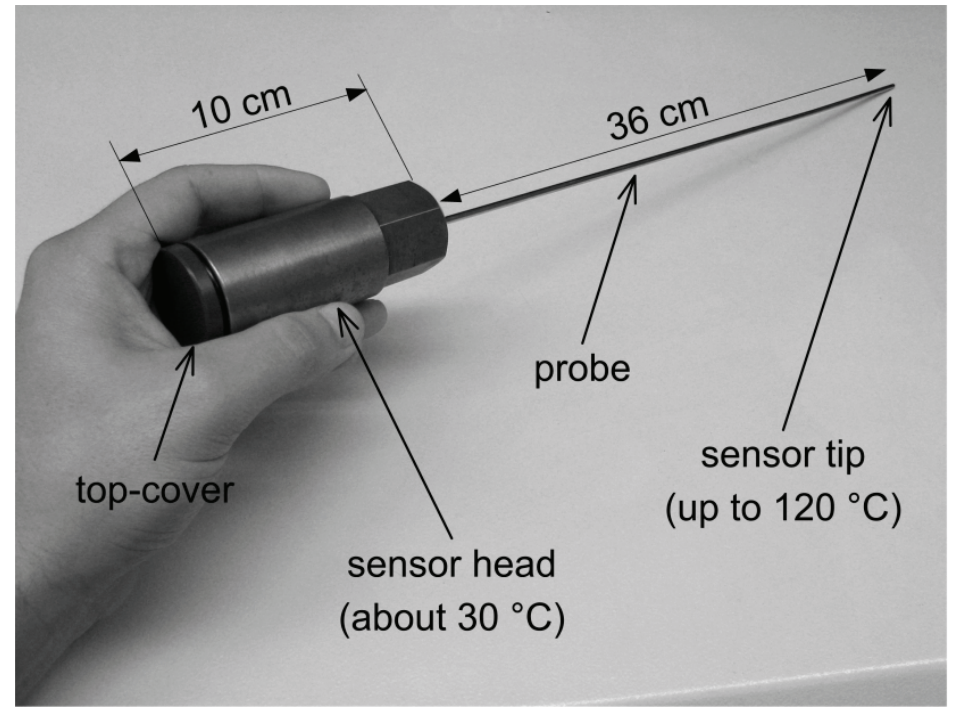

Fig. 6: Wireless sensor head with probe 


\section{Results}

The field tests with the battery powered module show that an installation and exchange of the wireless sensor could be made quickly with little effort. The radio signal was transmitted without difficulties at a distance of $3 \mathrm{~m}$ between sensor and receiver. The temperature changes of the inner side of the mold were displayed during the casting process in real time on the computer. It has been shown that the electronics are well protected by the sealed housing against splash water and dirt.

Up to now the thermoelectric powered module has only been tested in the laboratory. As expected the structural components (required for the mechanical stability) between the hot and cold side turned out to be heat bridges and thus reduced the effectiveness of the module. As shown

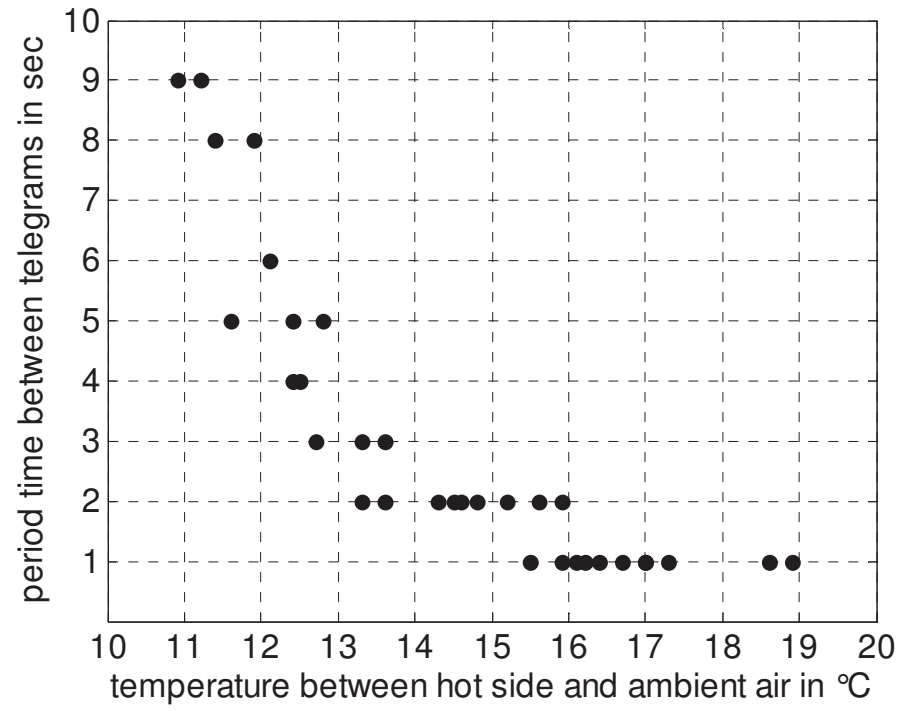

Fig. 7: Transmission cycle at natural convection in Fig. 7, the first radio telegrams are sent at a temperature difference of $11^{\circ} \mathrm{C}$ between the hot side of the sensor head and the ambient air. The maximum transmission rate of one measurement per second was observed at a temperature difference of $16{ }^{\circ} \mathrm{C}$. Experiments show, that an additional air flow can increase the thermal conductivity of the heat sink drastically.

\section{Conclusion}

The results of our investigations show that it is possible to exchange the existing wired measuring systems by wireless sensors without constructional modification of the considered types of ingot molds. This easy way of integration can immensely reduce the costs during the introduction of the wireless sensors. Also the quick and easy installation during reconstruction and maintenance of the molds leads to enormous economy of time and money. The sensor itself becomes a completely maintenance-free unit by using a self-sufficient thermoelectric power source.

The field tests have also shown that temperature changes during the casting process can be displayed in real time. Thus, the temperature data are available in the usual way to control the casting process. Furthermore, the robust industrial-suited sensor design raises the user acceptance by increasing the reliability.

Further field tests have to verify the performance and behavior of the thermoelectric powered prototype. The obtained results will establish a basis for building up a sensor network.

\section{Literature}

[1] Langer, M.; Arzberger, M.: Neue Wege bei der Durchbruchfrüherkennung beim Stranggiessen. Newsletter der SMS Demag AG. Zentralbereich Automation und Systemtechnik. Düsseldorf 2009.

[2] Dalola, Simone, et al.: Characterization of Thermoelectric Modules for Powering Autonomous Sensors. IEEE Transactions on Instrumentation and Measurement. 58, Januar 2009, Bd. 1.

[3] Mamaschew, S.: Entwicklung, Erprobung und Aufbau eines energieautarken drahtlosen Temperatursensors für industrielle Anwendungen. Technische Universität Clausthal, 2009.

[4] Kimball, J. W., Flowers, T. L. und Chapman, P. L.: Issues with Low-Input-Voltage Boost Converter Design. Grainger Center for Electric Machinery and Electromechanics, University of Illinois.

[5] Schmidt, F.; Heller, W.; Rahusen, D.; Sikora, A.: Routing-Protokoll für komplexe energieautarke Funknetze. MikroSystemTechnik Kongress 2009. Berlin, 2009. 